\section{Multiple Strictures in Jejunal \\ Crohn's Disease: Push \\ Enteroscopy Dilation}

Therapeutic push enteroscopy (TPE) is useful when bleeding from small bowel lesions occurs and endoscopic access is difficult. We have reported previously that push enteroscopy is useful in diagnosis of Crohn's disease (CD) without macroscopic lesions in the colon and upper digestive tract, because macroscopic and microscopic findings [1]. Complications in the proximal small bowel are rare, but in some cases surgery in duodenal and jejunal stenosis requires intestinal derivation and changes in their medical approach. Endoscopic dilation of stenosis in CD is a good treatment in the colon and upper digestive tract. We report an alternative technique by TPE in the jejunum.

A 37-year-old man with CD suffered from vomiting and gastric pain without inflammatory disease activity. The findings of upper endoscopy as far as the Treitz angle were normal, but there was food retention. Enteroclysis revealed multiple stenoses in the jejunum and upper digestive subobstruction. Colonoscopy with ileoscopy showed normal findings. The patient underwent TPE (Fuji video enteroscope 200 WM) and multiple fibrous stenoses in the proximal jejunum were confirmed 10 $40 \mathrm{~cm}$ below the Treitz angle (Figures 1,2). We then used a special long (3500 mm) balloon dilator (Olympus, Medical Europa, Barcelona, Spain) with filled contrast over a 0.035 -inch guide wire to make successive dilations of all the strictures (Figure 3). The balloon was inflated up to $2 \mathrm{~cm}$ over 2 minutes. Finally, the enteroscope was passed through the distal jejunum. The jejunal biopsies taken after dilation revealed CD without malignancy. After 3 weeks, we carried out another dilation, and 18 months later, the patient was in an optimal state of health.

In the upper digestive tract repeated dilations are successful in CD in continuing to prevent the need for surgery if the dilation reaches up to $2 \mathrm{~cm}$ in order to prevent recurrence [2]. The use of combined radiology [3] is important for the evaluation of morphological aspects before and after dilation. The edema or fibrous component of the stricture is the main factor to explain recurrences and in some cases two or more sessions are needed. The dilation procedure with TPE in small-bowel stenosis is quick, relatively simple, and within reach of most endoscopists; moreover, its toler-

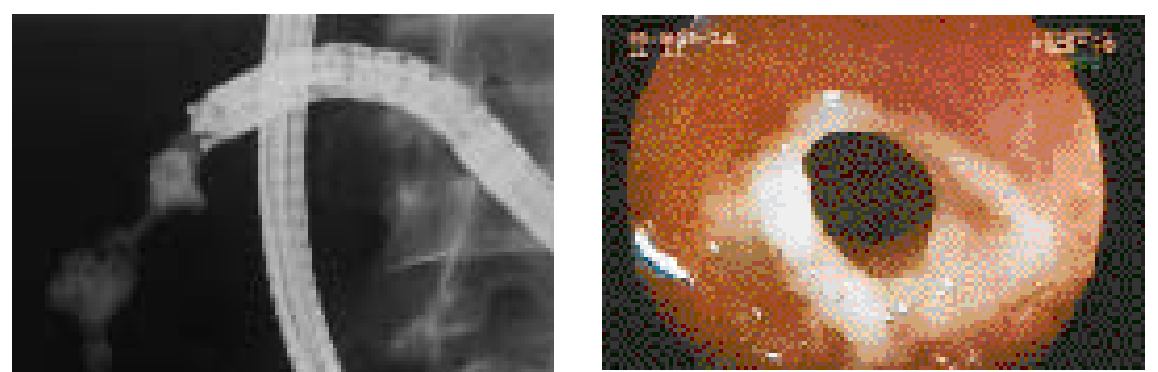

Figure 2 Enteroscopic view of jejunal stenosis in Crohn's disease (CD)

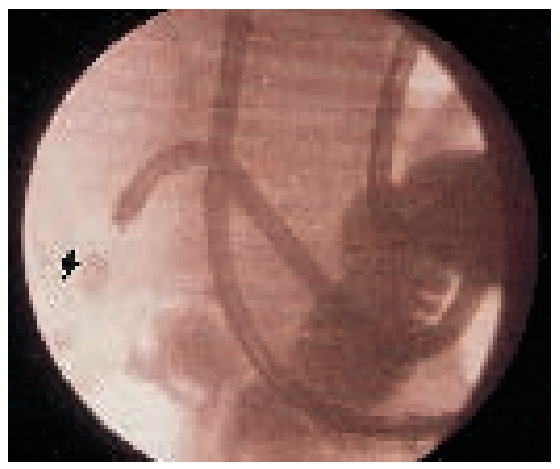

Figure 1 Multiple jejunal stenosis in oral push enteroscopy

Figure 3 Balloon dilation in the jejunum (arrow)

ance is acceptable. The possibility of associated malignancy is also evaluated. To our knowledge, this is the first report which describes the usefulness of TPE in the dilation of jejunal CD.

\section{E. Pérez-Cuadrado, E. Molina Pérez}

Division of Gastroenterology,

Endoscopy Unit, Naval Hospital,

La Coruña, Spain

\section{References}

${ }^{1}$ Pèrez-Cuadrado E, Macenlle R, Iglesias $\mathrm{J}$, et al. Usefulness of oral video push enteroscopy in Crohn's disease. Endoscopy 1997; 29: 745-747

${ }^{2}$ Matsui T, Hatakeyama S, Ikeda K. Longterm outcome of endoscopic balloon dilation in obstructive gastroduodenal Crohn's disease. Endoscopy 1997; 29: 640-645
${ }^{3}$ Pèrez-Cuadrado E, Lamas D, Robles A. Videoenteroscopia oral: estudio prospectivo sobre 30 casos. Rev Esp Enferm Dig 1996; 88: 9-15

Corresponding Author

E. Pèrez-Cuadrado, M.D.

Division of Gastroenterology

Endoscopy Unit

Naval Hospital

San Pedro de Leixa s/n

15405 Ferro

La Coruña

Spain

Fax: +34-8-198-1336308

E-mail: eperezcuadradom@meditex.es 